\title{
Characteristic of Aromatic Amino Acid Substitution at $\alpha 96$ of Hemoglobin
}

\author{
Jong-Whan Choi, Jong Hyuk Leeł, Kwang Ho Lee», Hyean-Woo Lee, Joon Hyung Sohn, \\ Joon Ho Yoon, Byung-Il Yeh, Seung Kyu Park, Kyu Jae Lee and Hyun-Won Kim* \\ Department of Biochemistry, 'Department of Opthalmology, "Department of Anesthesiology and Pain Medicine, \\ Department of Parasitology and Institute of Basic Medical Sciences, Yonsei University Wonju College of Medicine, Wonju 220-701, Korea
}

Received 27 April 2004, Accepted 10 September 2004

Replacement of valine by tryptophan or tyrosine at position $\alpha 96$ of the $\alpha$ chain ( $\alpha 96$ Val), located in the $\alpha_{1} \beta_{2}$ subunit interface of hemoglobin leads to low oxygen affinity hemoglobin, and has been suggested to be due to the extra stability introduced by an aromatic amino acid at the $\alpha 96$ position. The characteristic of aromatic amino acid substitution at the $\alpha 96$ of hemoglobin has been further investigated by producing double mutant $r \mathrm{Hb}(\alpha 42 \mathrm{Tyr} \rightarrow$ Phe, $\alpha 96$ Val $\rightarrow$ Trp). r Hb $(\alpha 42$ Tyr $\rightarrow$ Phe $)$ is known to exhibit almost no cooperativity in binding oxygen, and possesses high oxygen affinity due to the disruption of the hydrogen bond between $\alpha 42$ Tyr and $\beta 99$ Asp in the $\alpha_{1} \beta_{2}$ subunit interface of deoxy $\mathrm{Hb} \mathrm{A}$. The second mutation, $\alpha 96 \mathrm{Val} \rightarrow$ Trp, may compensate the functional defects of $r$ $\mathrm{Hb}(\alpha 42 \mathrm{Tyr} \rightarrow$ Phe), if the stability due to the introduction of trypophan at the $\alpha 96$ position is strong enough to overcome the defect of $r \mathrm{Hb}(\alpha 42 \mathrm{Tyr} \rightarrow$ Phe $)$. Double mutant $r$ Hb $(\alpha 42$ Tyr $\rightarrow$ Phe, $\alpha 96$ Val $\rightarrow$ Trp $)$ exhibited almost no cooperativity in binding oxygen and possessed high oxygen affinity, similarly to that of $r \mathrm{Hb}$ $\left(\alpha 42 \mathrm{Tyr} \rightarrow\right.$ Phe). ${ }^{1} \mathrm{H}$ NMR spectroscopic data of $r \mathrm{Hb}$ $(\alpha 42$ Tyr $\rightarrow$ Phe, $\alpha 96$ Val $\rightarrow$ Trp) also showed a very unstable deoxy-quaternary structure. The present investigation has demonstrated that the presence of the crucible hydrogen bond between $\alpha 42$ Tyr and $\beta 99 A s p$ is essential for the novel oxygen binding properties of deoxy $\mathrm{Hb}(\alpha 96 \mathrm{Val} \rightarrow \mathrm{Trp})$.

Keywords: Escherichia coli, ${ }^{1} \mathrm{H}$ NMR, Interfacial hydrogen bonding, Low oxygen affinity, Recombinant mutant hemoglobin

\section{Introduction}

Low oxygen affinity recombinant (r) $\mathrm{Hb}(\alpha 96 \mathrm{Val} \rightarrow \mathrm{Trp})$ and r Hb ( $\alpha 96 \mathrm{Val} \rightarrow \mathrm{Tyr}$ ) have been produced (Kim et al., 1995; Choi et al., 1998) using an Escherichia coli expression plasmid in which synthetic human $\alpha$ - and $\beta$-globin genes were coexpressed with the Escherichia coli methionine aminopeptidase gene. These artificial hemoglobins show low oxygen affinity, but high cooperativity in oxygen binding, and exhibit no unusual subunit dissociation when ligated. These novel properties provide an opportunity as a potential candidate for hemoglobin-based blood substitute.

Despite the replacement of a small amino acid residue, valine, by a large aromatic amino acid residue, both $\mathrm{r} \mathrm{Hb}$ $(\alpha 96 \mathrm{Val} \rightarrow$ Trp) and $\mathrm{r} \mathrm{Hb}(\alpha 96 \mathrm{Val} \rightarrow$ Tyr $)$ show very similar tertiary structures around the heme pockets and quaternary structures in the $\alpha_{1} \beta_{2}$ subunit interface compared to those of human normal adult hemoglobin (Kim et al., 1995; Choi et $a l .$, 1998). Another unique feature of this hemoglobin is that the ligated form, e.g. carbonmonoxy form, in its oxyquaternary structure can be converted to the deoxy-like quaternary structure by the addition of an allosteric effector, inositol hexaphosphate (IHP), without changing its ligation state, suggesting a very stable deoxy quaternary structure.

Recent MD simulation, using whole hemoglobin coordinates and x-ray crystallography of $\mathrm{r} \mathrm{Hb}(\alpha 96 \mathrm{Val} \rightarrow \mathrm{Trp})$, revealed that the indole side chain of tryptophan is directed toward the central cavity (Puise et al., 1998). In the structure, the indole nitrogen makes water mediated hydrogen bonds with $\beta 101 G$ lu, and was proposed as the structural basis for the low oxygen affinity of the $\mathrm{r} \mathrm{Hb}(\alpha 96 \mathrm{Val} \rightarrow \mathrm{Trp})$.

X-ray crystallographic studies of deoxy-Hb A show that $\beta 99$ Asp is hydrogen-bonded to both $\alpha 42$ Tyr and $\alpha 97$ Asn in the $\alpha_{1} \beta_{2}$ (or $\alpha_{2} \beta_{1}$ ) interface of deoxy-Hb A (Fermi et al., 1984 ), suggesting that the essential role of $\beta 99 A s p$ is to stabilize the deoxy- $\mathrm{Hb}$ molecule by making intersubunit hydrogen bonds; and thus, could provide the free energy of cooperativity in binding oxygen. Recent studies using site directed mutagenesis have shown that the hydrogen bond
*To whom correspondence should be addressed

E-mail: kimhwbio@wonju.yonsei.ac.kr 
between $\alpha 42$ Tyr and $\beta 99$ Asp plays a key role in stabilizing the deoxy quaternary structure (Imai et al., 1991; Kim et al., 1994), while the hydrogen bond between $\alpha 97$ Asn and 399 Asp has only a supporting role (Kim et al., 1996).

In the present work, a unique feature of the tryptophan side chain at $\alpha 96$ position has been further investigated by producing double mutant $\mathrm{r} \mathrm{Hb}(\alpha 42 \mathrm{Tyr} \rightarrow$ Phe, $\alpha 96 \mathrm{Val} \rightarrow \mathrm{Trp})$ using the E. coli expression system. $\mathrm{r} \mathrm{Hb}(\alpha 42 \mathrm{Tyr} \rightarrow \mathrm{Phe})$ is known to exhibit almost no cooperativity in binding oxygen, and possesses high oxygen affinity due to the missing crucial hydrogen bond between $\alpha 42$ Tyr and $\beta$ 99Asp (Imai et al., $1991)$. r Hb ( $\alpha 96$ Val $\rightarrow$ Trp $)$ has a very stable deoxy quaternary structure and shows low oxygen affinity. Thus, a $\alpha 96$ ValTrp substitution is expected to compensate the functional defects of $\mathrm{r} \mathrm{Hb}(\alpha 42 \mathrm{Tyr} \rightarrow \mathrm{Phe})$. The oxygen binding properties of $\mathrm{r} \mathrm{Hb}(\alpha 42 \mathrm{Tyr} \rightarrow \mathrm{Phe}, \alpha 96 \mathrm{Val} \rightarrow \mathrm{Trp})$ have been determined and ${ }^{1} \mathrm{H}$ NMR spectroscopy used to investigate the tertiary and quaternary structures.

\section{Materials and Methods}

Production of mutant $\mathbf{r} \mathbf{H b}$ The $\mathrm{Hb}$ A expression plasmid, PHE2, containing synthetic $\alpha$ and $\beta$-globin genes, and the $E$. coli methionine aminopeptidase gene were used to produce mutant $\mathrm{Hbs}$. The phagemid pTZ18U and E. coli JM109 were purchased form Bio-Rad and Promega, respectively. Synthetic human $\alpha$ - and $\beta$ globin genes were inserted into phagemid pTZ18U. Site directed mutagenesis was performed, as previously described (Shen et al., 1993). Synthetic oligonucleotide 5'-CGGAAGTCGAAATTAGTG GTC-3' and 5'-TTTGAAGTTCCATGGATCAAC-3' were used for the mutations, $\alpha 42$ TyrPhe and $\alpha 96$ ValTrp, respectively. The normal human $\alpha$ - and $\beta$-globin genes in plasmid pHE2 were then replaced by the mutated globin genes.

Growth and purification of $\mathbf{r} \mathbf{H b}$ The resulting plasmid was transformed into E. coli JM109, and the cells grown in TB medium in a 10-liter Microferm fermentor (New Brunswick Scientific, model BioFlo 3000$)$ at $30^{\circ} \mathrm{C}$ until the optical density at $600 \mathrm{~nm}$ reached 10 . The expression of $\mathrm{r} \mathrm{Hb}(\alpha 42 \mathrm{Tyr} \rightarrow \mathrm{Phe}, \alpha 96 \mathrm{Val} \rightarrow$ Trp) was induced by adding isopropyl $\beta$-thiogalactopyranoside to a concentration of $0.2 \mathrm{mM}$. The culture was then supplemented with hemin $(50 \mathrm{mg} / \mathrm{L})$, and the growth continued for at least another $4 \mathrm{~h}$. The cells were harvested by centrifugation and stored at -80 until needed for purification. The $\mathrm{r} \mathrm{Hb}(\alpha 42 \mathrm{Tyr} \rightarrow \mathrm{Phe}, \alpha 96 \mathrm{Val} \rightarrow \mathrm{Trp})$ was purified as previously described (Kim et al., 1996). Two columns were used in the final purification process: (i) a QSepharose fast-flow column (Pharmacia anion exchanger) to bind $\mathrm{Hb}$. After the sample had been loaded onto the column, it was thoroughly washed with the running buffer $(20 \mathrm{mM}$ Tris- $\mathrm{HCl} / 0.1$ mM EDTA at $\mathrm{pH}$ 8.3). The $\mathrm{Hb}$ fraction was then oxidized and reduced, as described in Shen et al. (1993). (ii) a Mono S column (Pharmacia cation exchanger HR16/10), with a gradient of $10 \mathrm{mM}$ sodium phosphate/0.1 mM EDTA at $\mathrm{pH} 6.8$ to $20 \mathrm{mM}$ sodium phosphate/0.1 mM EDTA at $\mathrm{pH} 8.3$ to purify the $\mathrm{r} \mathrm{Hb}(\alpha 42 \mathrm{Tyr} \rightarrow$ Phe, $\alpha 96$ Val $\rightarrow$ Trp).
Analytical procedures The mass spectrometric analyses were performed on a VG Quatttro-BQ (Fissions Instruments, VG Biotech, Altrincham, UK), as previously described (Shen et al., 1993).

Oxygen binding of $\mathbf{H b}$ sample To measure the oxygen binding of the $\mathrm{r} \mathrm{Hb}(\alpha 42 \mathrm{Tyr} \rightarrow$ Phe, $\alpha 96 \mathrm{Val} \rightarrow \mathrm{Trp})$ and $\mathrm{Hb} \mathrm{A}(0.1 \mathrm{mM}$ of each), oxygen-dissociation curves were measured by a Hemoxanalyzer (TCS Medical Products, Huntington Valley, PA) at $29^{\circ} \mathrm{C}$ in $0.1 \mathrm{M}$ sodium phosphate buffer. The partial $\mathrm{O}_{2}$ pressure at $50 \%$ saturation $\left(P_{50}\right)$ and the Hill coefficient $\left(n_{\max }\right)$ were determined from each curve.

NMR measurements ${ }^{1} \mathrm{H}-\mathrm{NMR}$ spectra were obtained on a Bruker AM-300 spectrometer, operating at $300 \mathrm{MHz}$ and $29^{\circ} \mathrm{C}$. All $\mathrm{Hb}$ samples were in $0.1 \mathrm{M}$ sodium phosphate buffer $(\mathrm{pH} 7.0)$ at concentrations of about $4 \%$. The water signal was suppressed by a jump-and-return pulse sequence (Plateau and Gueron, 1982). Typically 1024 scans were averaged to improve the signal-to-noise ratio. The proton chemical shifts are indirectly referenced to the methyl proton resonance of the sodium salt of 2, 2-dimethyl-2silapentane-5-sulfonate (DSS) using the water signal occurring 4.76 ppm downfield from that of DSS at $29^{\circ} \mathrm{C}$ as the internal reference.

\section{Results and Discussion}

The purification of $\mathrm{r} \mathrm{Hb}$ from $E$. coli cells generally produces several peaks on a Mono $\mathrm{S}$ column, with only showing the correct heme conformation. However, by oxidizing the $\mathrm{Hb}$ to the ferric state and then reducing it back to the ferrous state, and finally converting it back to either the $\mathrm{CO}$ or oxy form, an incorrectly inserted heme can be converted to the correct conformation (Shen et al., 1993; Kim et al., 1995). In the present investigation, $\mathrm{r} \mathrm{Hb}(\alpha 42 \mathrm{Tyr} \rightarrow \mathrm{Phe}, \alpha 96 \mathrm{Val} \rightarrow \mathrm{Trp})$ was oxidized and reduced to the $\mathrm{CO}$ form before its application to the Mono S column. The $\mathrm{r} \mathrm{Hb}(\alpha 42 \mathrm{Tyr} \rightarrow \mathrm{Phe}$, $\alpha 96$ Val $\rightarrow$ Trp) purified from E. coli JM109 showed two major peaks on Mono S column chromatography. Both $\mathrm{r} \mathrm{Hb}$ $(\alpha 42 \mathrm{Tyr} \rightarrow$ Phe, $\alpha 96 \mathrm{Val} \rightarrow$ Trp) peaks from the Mono $\mathrm{S}$ column showed a correctly inserted heme conformation by ${ }^{1} \mathrm{H}$ NMR spectroscopy (results not shown), but differed in their $\mathrm{N}$-terminal methionine contents. Mass spectrometric analyses of this purified $\mathrm{r} \mathrm{Hb}(\alpha 42 \mathrm{Tyr} \rightarrow$ Phe, $\alpha 96 \mathrm{Val} \rightarrow \mathrm{Trp})$ show that $47 \%$ of the combined $\alpha$ and $\beta$ chains for the first peak contain N-terminally added methionine, whereas only $7 \%$ of the total $\alpha$ and $\beta$ chains for the second peak contain $\mathrm{N}$-terminal methionine. Thus, the $\mathrm{r} \mathrm{Hb}(\alpha 42 \mathrm{Tyr} \rightarrow \mathrm{Phe}, \alpha 96 \mathrm{Val} \rightarrow \mathrm{Trp})$ from the second peak was used for further experiments.

The oxygen binding properties of $\mathrm{r} \mathrm{Hb}(\alpha 42 \mathrm{Tyr} \rightarrow \mathrm{Phe}$, $\alpha 96 \mathrm{Val} \rightarrow \operatorname{Trp}), \mathrm{Hb} \mathrm{A}$ and other mutant Hbs are compared in Table 1 . In $0.1 \mathrm{mM}$ sodium phosphate at $29^{\circ} \mathrm{C}, \mathrm{r} \mathrm{Hb}(\alpha 42 \mathrm{Tyr}$ $\rightarrow$ Phe, $\alpha 96$ Val $\rightarrow$ Trp) exhibits higher oxygen affinity than $\mathrm{Hb} \mathrm{A}$ and very low cooperativity in binding oxygen at neutral $\mathrm{pH}$. The addition of IHP to $\mathrm{r} \mathrm{Hb}(\alpha 42 \mathrm{Tyr} \rightarrow \mathrm{Phe}, \alpha 96 \mathrm{Val} \rightarrow$ Trp) caused only slight changes in both oxygen affinity and cooperativity. These oxygen binding properties of $r \mathrm{Hb}$ 
Table 1. $\mathrm{P}_{50}$ and $n_{\max }$ values of $\mathrm{Hb} \mathrm{A}$ and $\mathrm{r} \mathrm{Hbs}$ in $0.1 \mathrm{M}$ Phosphate at $\mathrm{pH} 7.4$ and 29

\begin{tabular}{|c|c|c|c|c|c|}
\hline \multirow{2}{*}{$\mathrm{Hb}$} & \multicolumn{2}{|c|}{$P_{50}, \mathrm{mmHg}$} & \multicolumn{2}{|c|}{$n_{\max }$} & \multirow{2}{*}{ Reference } \\
\hline & -IHP & $+\mathrm{IHP}^{\mathrm{a}}$ & $-\mathrm{IHP}$ & $+\mathrm{IHP}^{\mathrm{a}}$ & \\
\hline $\mathrm{Hb} \mathrm{A}$ & 8.0 & 35.5 & 3.1 & 2.6 & present work \\
\hline r $\mathrm{Hb}(\mathrm{a} 96 \mathrm{Val} \rightarrow \mathrm{Trp})$ & 11.6 & $\mathrm{~b}$ & 2.6 & $\mathrm{~b}$ & Kim et al (1995) \\
\hline r Hb $(\alpha 97$ Asn $\rightarrow$ Ala $)$ & 1.1 & 5.2 & 1.4 & 2.6 & Kim et al (1996) \\
\hline r Hb $(\alpha 42 \mathrm{Tyr} \rightarrow$ Asp, $\beta 99 \mathrm{Asp} \rightarrow$ Asn $)$ & 1.9 & 10.0 & 1.7 & 2.4 & Kim et al (1994) \\
\hline r Hb $(\alpha 42 \mathrm{Tyr} \rightarrow \mathrm{His})^{\mathrm{c}} 1.4$ & 15 & 1.9 & 2.1 & & Imai et al (1991) \\
\hline r Hb $(\alpha 42 \mathrm{Tyr} \rightarrow \mathrm{Phe})^{\mathrm{c}}$ & 0.6 & 1.0 & 1.1 & 1.4 & Imai et al (1991) \\
\hline Hb Kempsey $(\beta 99 A s p \rightarrow A s n)^{c}$ & 0.2 & $1.1^{\mathrm{d}}$ & 1.1 & $1.7^{\mathrm{d}}$ & Bunn et al (1974) \\
\hline r Hb $(\alpha 42 \mathrm{Tyr} \rightarrow \mathrm{Phe}, \alpha 96 \mathrm{Val} \rightarrow \operatorname{Trp})$ & 1.2 & 1.7 & 1.1 & 1.4 & present work \\
\hline
\end{tabular}

${ }^{a} \mathrm{IHP}$ concentration was $2 \mathrm{mM}$ unless otherwise specified.

${ }^{b}$ Not measured.

'In $0.05 \mathrm{M}$ Bis-Tris (pH 7.4) containing $0.1 \mathrm{M} \mathrm{Cl}^{-}$at $25^{\circ} \mathrm{C}$.

${ }^{\mathrm{d}}$ In $0.01 \mathrm{M}$ Bis-Tris (pH 7.2) containing $0.1 \mathrm{M} \mathrm{Cl}^{-}$at $20^{\circ} \mathrm{C}$.

${ }^{\mathrm{e}} \mathrm{IHP}$ concentration was $1 \mathrm{mM}$.

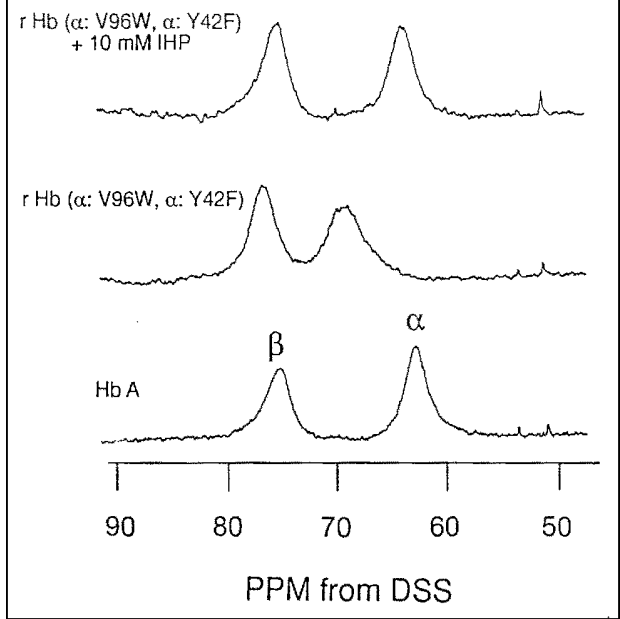

Fig. 1. 300-MHz Hyperfine-shifted $\mathrm{N}_{\delta} \mathrm{H}$ exchangeable proton resonances of the proximal histidine residues of deoxy $\mathrm{r} \mathrm{Hb}$ $(\alpha 42 \mathrm{Tyr} \rightarrow \mathrm{Phe}, \alpha 96 \mathrm{Val} \rightarrow \mathrm{Trp})$ and deoxy $\mathrm{Hb} \mathrm{A}$ in $0.1 \mathrm{M}$ phosphate in $\mathrm{H}_{2} \mathrm{O}$ at $\mathrm{pH} 7.0$ and $29^{\circ} \mathrm{C}$.

$(\alpha 42 \mathrm{Tyr} \rightarrow$ Phe, $\alpha 96 \mathrm{Val} \rightarrow$ Trp) are rather similar to those of $\mathrm{Hb}$ Kempsey and $\mathrm{r} \mathrm{Hb}(\alpha 42 \mathrm{TyrPhe})$, which are known to have no hydrogen bond in the $\alpha_{1} \beta_{2}$ subunit interface of their deoxy forms, compared to those of $\mathrm{r} \mathrm{Hb}(\alpha 42 \mathrm{Tyr} \rightarrow \mathrm{His})($ Imai et al., 1991), r Hb( $\alpha 97 \mathrm{Asp} \rightarrow \mathrm{Ala})(\mathrm{Kim}$ et al., 1996) and r Hb $(\alpha 42 \mathrm{Tyr} \rightarrow$ Asp, $\beta 99$ Asp $\rightarrow$ Asn)(Kim et al., 1994), which are believed to have intermediate strength hydrogen bonds in the $\alpha_{1} \beta_{2}$ ?subunit interface of their deoxy forms.

${ }^{1} \mathrm{H}$ NMR spectroscopy has been shown to be an excellent tool to investigate the tertiary and quaternary structural features of $\mathrm{Hb}$ (Ho, 1992). Very low-field ${ }^{1} \mathrm{H}$ resonances of $\mathrm{Hb} \mathrm{A}$ and $\mathrm{r}$ $\mathrm{Hb}(\alpha 42 \mathrm{Tyr} \rightarrow$ Phe, $\alpha 96 \mathrm{Val} \rightarrow \mathrm{Trp})$ are compared in Fig. 1. The resonance at $\sim 63 \mathrm{ppm}$ from DSS has been assigned to the hyperfine-shifted $\mathrm{N}_{\delta} \mathrm{H}$ exchangeable proton of the proximal histidine residue ( $\alpha 87 \mathrm{His}$ ) of the $\alpha$ chain of the deoxy-Hb A,

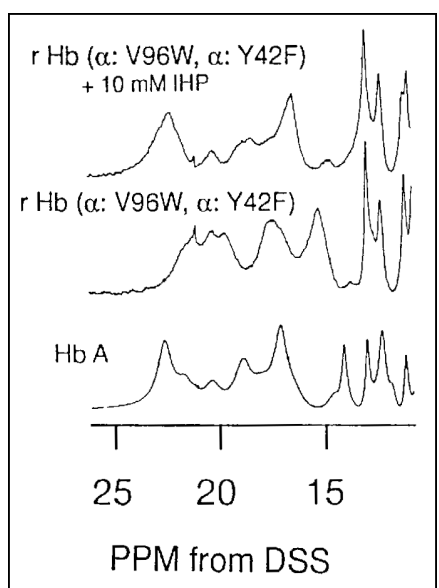

Fig. 2. 300-MHz Hyperfine-shifted and exchangeable proton resonances of deoxy $\mathrm{r} \mathrm{Hb}(\alpha 42 \mathrm{Tyr} \rightarrow \mathrm{Phe}, \alpha 96 \mathrm{Val} \rightarrow \mathrm{Tr} p)$ and deoxy $\mathrm{Hb} \mathrm{A}$ in $0.1 \mathrm{M}$ phosphate in $\mathrm{H}_{2} \mathrm{O}$ at $\mathrm{pH} 7.0$ and $29^{\circ} \mathrm{C}$. The spike at $21 \mathrm{ppm}$ was due to an instrumental artifact.

and that at $\sim 77 \mathrm{ppm}$ to the corresponding residue of the $\beta$ chain ( $\beta 92 \mathrm{His})$ of deoxy-Hb A (Takahashi et al., 1980; La Mar et al., 1980). The chemical shift positions of these two proximal histidyl resonances in $\mathrm{r}$ deoxy-Hb $(\alpha 42 \mathrm{Tyr} \rightarrow \mathrm{Phe}, \alpha 96 \mathrm{Val}$ $\rightarrow$ Trp) have been down-field shifted. However, the addition of IHP could convert the spectrum to one similar to that of deoxy$\mathrm{Hb} \mathrm{A}$. This is a typical feature of mutant $\mathrm{Hbs}$ missing the crucial hydrogen bond between $\alpha 42$ Tyr and $\beta 99$ Asp at the $\alpha_{1} \beta_{2}$ subunit interface.

The exchangeable and ferrous hyperfine-shifted proton resonances of $\mathrm{r} \mathrm{Hb}(\alpha 42 \mathrm{Tyr} \rightarrow \mathrm{Phe}, \alpha 96 \mathrm{Val} \rightarrow \mathrm{Trp})$ in the deoxy form are shown in Fig. 2. The resonance at $\sim 14 \mathrm{ppm}$ from DSS of the $\mathrm{Hb} \mathrm{A}$ has been assigned to the intersubunit hydrogen bond between $\alpha 42$ Tyr and $\beta 99$ Asp (Fung and Ho, 1975), and is a key marker for the deoxy-quaternary structure of $\mathrm{Hb} \mathrm{A}$. This resonance is completely absent from the spectra 
of $\mathrm{r} \mathrm{Hb}(\alpha 42 \mathrm{Tyr} \rightarrow \mathrm{Phe}, \alpha 96 \mathrm{Val} \rightarrow \mathrm{Trp})$, and no new noticeable exchangeable resonance appeared. All the other exchangeable resonances assigned to the $\alpha_{1} \beta_{2}$ interfacial hydrogen bonds between the $\alpha 126 \mathrm{Asp}$ and $\beta 35 \mathrm{Tyr}(\sim 12.9 \mathrm{ppm})$ and between the $\alpha 103 \rightarrow$ Asp and $\beta 108 \rightarrow$ Asn $(\sim 12.1$ ppm) (Russu et al., 1987) were present in the ${ }^{1} \mathrm{H}$ NMR spectrum of $\mathrm{r} \mathrm{Hb}$ $(\alpha 42 \mathrm{Tyr} \rightarrow$ Phe, $\alpha 96 \mathrm{Val} \rightarrow$ Trp). The hyperfine-shifted resonances arise from the protons on the heme groups and their nearby amino acid residues due to the hyperfine interactions between protons and the unpaired electrons of $\mathrm{Fe}(\mathrm{II})$ in the heme iron atoms. The hyperfine-shifted resonances of $\mathrm{r} \mathrm{Hb}(\alpha 42 \mathrm{Tyr} \rightarrow$ Asp, $\beta 99$ Asp $\rightarrow$ Asn) over the spectral region $\sim 16$ to $\sim 24 \mathrm{ppm}$ showed significant changes from those of $\mathrm{Hb} \mathrm{A}$. However, the addition of IHP can convert the spectrum to one similar to that of deoxy-Hb form. These results indicate that the deoxy form of $\mathrm{r} \mathrm{Hb}(\alpha 42 \mathrm{Tyr} \rightarrow$ Phe, $\alpha 96 \mathrm{Val} \rightarrow \mathrm{Trp})$ is very unstable i.e., exists in an oxy-like (or R type) quaternary structure. Mutant Hbs, which are known to have intermediate strength hydrogen bonds in the $\alpha_{1} \beta_{2}$ subunit interface, such as, $\mathrm{r} \mathrm{Hb}(\alpha 42 \mathrm{Tyr} \rightarrow$ His) (Imai et al., 1991) and r Hb ( $\alpha 97 \mathrm{Asn} \rightarrow \mathrm{Ala})$ (Kim et al., 1996), all exhibited stable deoxy-like quaternary structures from the ${ }^{1} \mathrm{H}-\mathrm{NMR}$ spectra.

Double-mutant recombinant hemoglobin, $\mathrm{r} \mathrm{Hb}(\alpha 42 \mathrm{Tyr} \rightarrow$ Asp, $\beta 99$ Asp $\rightarrow$ Asn), was recently produced by site-directed mutagenesis, in an expectation that a new hydrogen bond involving $\beta 99$ Asn could be induced by replacing $\alpha 42$ Tyr by a strong hydrogen bond acceptor, such as Asp (Kim et al., 1994; Yeh et al.,1998). A modified $\mathrm{Hb}$ was expected to regain the cooperativites lost in $\mathrm{Hb}$ Kempsey ( $\beta 99 \mathrm{Asp} \rightarrow \mathrm{Asn}$ ). The oxygen affinity of $\mathrm{r} \mathrm{Hb}(\alpha 42 \mathrm{Tyr} \rightarrow$ Asp, $\beta 99$ Asp $\rightarrow$ Asn), while still high, was significantly lower than that of Hb Kempsey ( $\beta 99$ Asp $\rightarrow$ Asn), with the restoration of substantial cooperativity. The ${ }^{1} \mathrm{H}$ NMR spectrum of deoxy-r Hb ( $\alpha 42 \mathrm{Tyr} \rightarrow$ Asp, $\beta 99 \mathrm{Asp}$ $\rightarrow$ Asn) also showed a very stable new deoxy-like structure.

In the present investigation, $\mathrm{r} \mathrm{Hb}(\alpha 42 \mathrm{Tyr} \rightarrow \mathrm{Phe}, \alpha 96 \mathrm{Val}$ $\rightarrow$ Trp) has been produced with the expectation of compensating for the functional defects of $\mathrm{r} \mathrm{Hb}(\alpha 42 \mathrm{Tyr} \rightarrow$ Phe), if the $\alpha 96 \operatorname{Trp}$ should add extra stability to the deoxy structure of $\mathrm{Hb}(\alpha 42 \mathrm{Tyr} \rightarrow \mathrm{Phe})$, as suggested by Kim et al. (1995). However, neither the oxygen binding properties nor ${ }^{1} \mathrm{H}$ spectroscopic results of $\mathrm{r} \mathrm{Hb}(\alpha 42 \mathrm{Tyr} \rightarrow$ Phe, $\alpha 96 \mathrm{Val} \rightarrow \mathrm{Trp})$ in the present investigation showed the expected compensation.

These results could mean that the extra stability of the deoxy form introduced by $\alpha 96 \mathrm{Val} \rightarrow \operatorname{Trp}$, either due to the water mediated hydrogen bonds between the indole ring of tryptophan and $\beta 101 G l u$ (Puis et al., 1998), or due to the direction of the aromatic ring into the internal cavity (Kim et al., 2001; Choi et al., 1998), may not be strong enough to maintain the deoxy-like quaternary structure in the absence of the crucial hydrogen bond between $\alpha 42$ Tyr and $\beta 99$ Asp. The present investigation has demonstrated that the presence of the crucial hydrogen bond between $\alpha 42 \mathrm{Tyr}$ and $\beta 99 \mathrm{Asp}$ is essential for the novel oxygen binding properties of $\mathrm{r} \mathrm{Hb}$ $(\alpha 96$ Val $\rightarrow$ Trp).
Acknowledgments This research was supported by a research grant of the Yonsei University Wonju College of Medicine for 2002.

\section{References}

Bunn, H. F., Wohl, R. C., Bradley, T. b., Cooley, M. and Gibson, Q. H. (1974) Functional properties of hemoglobin Kempsey $J$. Biol. Chem. 249, 7402-7409.

Choi, J.-W., Yeh, B.-I., Han, D.-P., Lee, H.-W., Sohn, J. H., Jung, S. and Kim, H.-W. (1998) Mutant recombinant hemoglobin $(\alpha 96 \mathrm{Val} \rightarrow \mathrm{Tyr})$ exhibits low oxygen affinity and high cooperativity. J. Biochem. Mol. Biol. 31, 595-599.

Fermi, G., Perutz, M. F., Shaananm B. and Fourme, R. (1984) A crystal structure of human deoxyhemoglobin at $1.74 \AA$. J. Mol. Biol. 175, 159-174.

Fung, L. W.-M. and Ho, C. (1975) A proton nuclear magnetic resonance study of the quaternary structure of human hemoglobin in water. Biochemistry 14, 2526-2535.

Ho, C. (1992) Proton nuclear magnetic resonance studies on hemoglobin: cooperative interactions and partially ligated intermediates. Advan. Protein Chem. 43, 153-312.

Imai, K., Fushitani, K., Miyazaki, G., Ishimori, K., Kitagawa, T., Wads, Y., Morimoto, H., Morishima, I., Shih, D. T.-B. and Tame, J. (1991) Site-directed mutagenesis in haemoglobin: fuctional role of tyrosine-42(C7) $\alpha$ at the $\alpha_{1} \beta_{2}$ interface. J. Mol. Biol. 218, 769-778.

Kim, H.-W., Shen, T.-J., Sun, D. P., Ho, N. T., Madrid, M., Tam, M. F., Zou, M., Cottam, P. F. and Ho, C. (1994) Restoring allosterism with compensatory mutations in hemoglobins Proc. Nat. Sci. USA 82, 48.

Kim, H.-W., Shen, T.-J., Sun, D. P., Ho, N. T. Madrid, M. and Ho, C. (1995) A low oxygen affinity recombinant $\mathrm{Hb}(\alpha 96 \mathrm{Val}$ $\rightarrow$ Trp): switching quaternary structure without changing the ligation state. J. Mol. Biol. 248, 867-882.

Kim, H.-W., Shen, T.-J., Sun, D. P., Ho, N. T., Zou, M., Tam, M. T. and Ho, C. (1996) Contribution of asparagine at $\alpha 97$ to the cooperative oxygenation process of hemoglobin. Biochemistry 35, 6620-6627.

Kim, H.-W., Lee, C.-H., Jung, S. and Won, Y. (2001) Molecular dynamics simulations of a low oxygen affinity mutant hemoglobin. Bul. Kor. Chem. Soc. 22, 253.

La Mar, G. N., Nagai, K., Jue, T., Budd, D., Gersonde, K., Sick, H., Kagimoto, T., Hayashi, A. and Taketa, F. (1980) Assignment of proximal histidyl imidazole exchangeable proton resonances to individual subunits. Biochem. Biophys. Res. Commun. 96, 1172-1177.

Plateau, P. and Gueron, M. (1982) Exchangeable proton NMR without base-line distortion, using new strong-pulse sequence. J. Am. Chem. Soc. 104, 7310-7311.

Puis, Y. A., Zou, M., Ho, N. T., Ho, C. and Almo, S. C. (1998) Novel water-mediated hydrogen bonds as the structural basis for the low oxygen affinity of the blood substitute candidate $\mathrm{rHb}(\alpha 96$ Val $\rightarrow$ Trp $)$. Biochemistry 21, 9258-9265.

Russu, I. M., Ho, N. T. and Ho, C. (1987) Aproton nuclear Overhauser effect investigation of the subunit interfaces in human normal adult hemoglobin. Biochim. Biophys. Acta 914, 
$40-48$.

Shen, T.-J., Ho, N. T., Simplaceanu, V., Zou, M., Green, B. N., Tam, M. F. and Ho, C. (1993) Production of unmodified human adult hemoglobin in Escherichia coli. Proc. Natl. Acad. Sci. USA 90, 8108-8112.

Takahashi, S., Lim, A. K.-L. C. and Ho, C. (1980) Proton nuclear magnetic resonance studies of hemoglobins $\mathrm{M}$ Boston $\alpha(58 \mathrm{E} 7 \mathrm{His} \rightarrow \mathrm{Tyr}) \quad$ and $\mathrm{M} \quad$ Milwaukee $\quad \beta(67 \mathrm{E} 11 \mathrm{Val} \rightarrow \mathrm{Glu})$ : spectral assignment of hyperfine-shifted proton resonances and of proximal histidine(E7) $\mathrm{NH}$ resonances to the $\alpha$ and $\beta$ chains of normal human adult hemoglobin. Biochemistry 19, 51965202.

Yeh, B.-I., Choi, J.-W., Han, D.-P., Lee, H.-W., Sohn, J. H., Jung, S. and Kim, H.-W. (1998) Functional defects of $\mathrm{Hb}$ Kemsey $(\beta 99$ Asp $\rightarrow$ Asn) can be compensated by insertion of new interfacial hydrogen bond at the $\alpha_{1} \beta_{2}$ subunit interface. $J$. Biochem. Mol. Biol. 31, 590-594. 